\title{
Optical Bistability in a Two-State Dissipative Medium Inside a Fabry-Perot Cavity
}

\author{
M. M. EL-Nicklawy, A. F. Hassan, A. T. Matar and H. A. Hafez. \\ Physics Department, Faculty of Science, Helwan University, \\ Helwan, Cairo, Egypt.
}

\begin{abstract}
In this work a theoretical model treating the optical bistability in a two state dissipative atomic medium inside a Fabry-Perot cavity is presented. The model is based on a semiclassical point of view to explain the field-matter interaction. The atoms of the medium are described by the "quantum polarization equation of motion" while the field is described by the "classical Maxwell's field equations". Applying the boundary conditions to the fields inside the Fabry-Perot cavity, the occurrence of optical bistability is deduced between the incident field intensity and both the intracavity and transmitted field intensities. Also the effect of building standing and nonstanding waves inside the cavity on bistability is discussed.
\end{abstract}

\section{Introduction:}

An optical system which exhibits two steady transmission states for the same input intensity is said to be optically bistable [1]. Nonlinearity and feedback are the main features required for making a bistable device. The optical bistability (OB) can be extrinsic if an external feedback is present, as in experiments with cavities, or intrinsic when it occurs at the atomic scale [2]. Bistable devices are important in the digital circuits and in communications, signal processing and computing. They are used as switches, logic gates and memory elements (flip-flops) [3]. There is an increasing interest in new bistable systems with possible applications in optical network technology [4]. Bistable systems such as electro-optical, acoustic-optical, magneto-optical hybrid bistable systems and electromagnetically induced systems have been investigated in many works [1].

Many researches dealing with nonlinear optics studied the occurrence of (OB) through different view points. The semiclassical mean field of the (OB) has been developed [5-7]. The quantum statistical mean field theory, the numerical analysis in Fabry-Perot cavity, and the dynamics of a bistable system 
have been studied in [8-10], [11,12], and [5,6,13] respectively. The (OB) was handled through a pure interferometric, and semiclassical view points [14-16]. A nonlinear classical differential equation of motion and Maxwell's field equations were considered to illustrate the field-matter interaction [16]. The effect of the spectral profile of the incident field and its halfwidth on bistability was studied $[15,16]$.

In the present work the $(\mathrm{OB})$ is treated semiclassically through the quantum polarization equation of motion [17] and Maxwell's field equations. In the quantum polarization equation of motion the number of dipoles induced in the medium by the action of a given deriving field is considered to be the population difference between the two states of the medium as a quantum mechanical correction to the classical polarization equation of motion. For a dissipative medium inside a Fabty-Perot cavity the nonlinearity results in the absorption coefficient of the medium while the feed-back results from the boundary conditions of the cavity. We will see that the field dependence of the atomic population difference plays the most important role in the appearance of nonlinearity in the absorption coefficient.

\section{Theory.}

\subsection{The quantum polarization equation of motion.}

The quantum polarization equation of motion describing an electron oscillator with damping in a two state atomic system exerted by an external electric field is given by [17]:

$$
\frac{\partial^{2} p(z, t)}{\partial t^{2}}+2 \beta \frac{\partial p(z, t)}{\partial t}+\omega_{a}^{2} p(z, t)=\frac{\Delta N e^{2}}{m} E(z, t)
$$

where:

$p$ is the polarization density as a function of space $z$ and time $t$,

$\beta$ is a damping factor,

$\omega_{a}$ is the atomic resonance frequency,

$e$ and $m$ are the charge and mass of the electron respectively and

$E$ is the deriving field.

The response of the atomic polarization density $p$ in Eqn. (1) is proportional to the population difference density $\Delta N=N_{1}-N_{2}$ between the populations (atoms per unit volume) in the lower and upper levels of the atomic transition. The number $\Delta N$ is not actually constant but depends on the field intensity and on the period of interaction between the deriving field, and the atoms of the medium. 
If an external monochromatic field of the form $E(z, t)=\varepsilon(z) e^{-i\left(\omega t-k_{0} z\right)}$ exerts a two state medium, in which the upper state is initially empty, the atomic population $N_{2}$ of the upper state will be given by [18]:

$$
N_{2}=\frac{N R_{21}}{A_{21}+2 R_{21}}\left[1-e^{-\left(A_{21}+2 R_{21}\right) t}\right]
$$

with

$$
R_{21}=\sigma \Phi=\frac{|\mu \varepsilon(z) / \hbar|^{2} \beta / 2}{\left(\omega_{a}-\omega\right)^{2}+\beta^{2}}
$$

where:

$N$ is the total atomic population, equal to $N_{1}+N_{2}$ for the two state system,

$A_{21}$ is the spontaneous emission rate of an atom,

$R_{21}$ is the stimulated emission rate of an atom which depends on the field intensity,

$\sigma$ is the atomic absorption cross-section,

$\Phi$ is the photon flux (photons $/ \mathrm{cm}^{2}$.sec ) associated with the light field of complex amplitude $\varepsilon(z)$ and

$\mu$ is the projection of the dipole moment in the direction of $E(z, t)$.

From equation (2) the atomic population difference $\Delta N=N_{1}-N_{2}$ is given by:

$$
\begin{aligned}
\Delta N & =N_{1}-N_{2} \\
& =N-2 N_{2} \\
& =\frac{N}{A_{21}+2 R_{21}}\left[A_{21}+2 R_{21} e^{-\left(A_{21}+2 R_{21}\right) t}\right]
\end{aligned}
$$

For the field $E(z, t)=\varepsilon(z) e^{-i\left(\omega t-k_{0} z\right)}$, taking $\Delta N$ from Eqn. (4) for a steady state, i.e. $\Delta N=\frac{N A_{21}}{A_{21}+2 R_{21}}$, and substituting in Eqn. (1), we have:

$\frac{\partial^{2} p(z, t)}{\partial t^{2}}+2 \beta \frac{\partial p(z, t)}{\partial t}+\omega_{a}^{2} p(z, t)=\frac{\gamma N A_{21}}{A_{21}+2 R_{21}} \varepsilon(z) e^{-i\left(\omega t-k_{0} z\right)}$

where $\gamma=\frac{e^{2}}{m}$.

The steady state solution of Eqn. (5) is given by:

$$
p(z, t)=\rho(z) e^{-i\left(\omega t-k_{0} z\right)}
$$


where $\rho(z)=\frac{\gamma N A_{21}}{\left(A_{21}+2 R_{21}\right)\left[\left(\omega_{a}^{2}-\omega^{2}\right)^{2}+4 \beta^{2} \omega^{2}\right]^{1 / 2}} e^{i \theta} \varepsilon(z)$

The induced polarization and the deriving field are out of phase by a value $\theta$, given by :

$$
\begin{aligned}
& \sin \theta=\frac{2 \beta \omega}{\left[\left(\omega_{a}^{2}-\omega^{2}\right)^{2}+4 \beta^{2} \omega^{2}\right]^{1 / 2}} \\
& \cos \theta=\frac{\left(\omega_{a}^{2}-\omega^{2}\right)}{\left[\left(\omega_{a}^{2}-\omega^{2}\right)+4 \beta^{2} \omega^{2}\right]^{1 / 2}}
\end{aligned}
$$

\subsection{The field equations inside a Fabry-Perot cavity.}

Setting now the medium inside a Fabry-Perot cavity, assuming that the complex amplitude of the field $\varepsilon(z)$ varies slowly compared with the carrier wave $e^{-i\left(\omega t-k_{0} z\right)}$, this justifies inequalities such as [18]:

$|d \varepsilon(z) / d z|<<k|\varepsilon(z)|$ and $\quad\left|d^{2} \varepsilon(z) / d z^{2}\right|<<k|d \varepsilon(z) / d z|$

Considering these inequalities in the Maxwell's wave equation, the forward and the backward field equations inside the cavity can be given by:

$$
\begin{aligned}
& \frac{\partial E_{F}(z, t)}{\partial t}+C \frac{\partial E_{F}(z, t)}{\partial z}=\frac{i \omega}{2 \epsilon_{0}} P_{F}(z, t) \\
& \frac{\partial E_{B}(z, t)}{\partial t}-C \frac{\partial E_{B}(z, t)}{\partial z}=\frac{i \omega}{2 \epsilon_{0}} P_{B}(z, t)
\end{aligned}
$$

Subjected to the following boundary conditions

$$
\begin{aligned}
& \mathrm{E}_{\mathrm{F}}(0, \mathrm{t})=\sqrt{T} \mathrm{E}_{\mathrm{In}}(0, \mathrm{t})+\sqrt{R} \mathrm{E}_{\mathrm{B}}(0, \mathrm{t}) \\
& \mathrm{E}_{\mathrm{T}}(\mathrm{L}, \mathrm{t})=\sqrt{T} \mathrm{E}_{\mathrm{F}}(\mathrm{L}, \mathrm{t}) \\
& \mathrm{E}_{\mathrm{B}}(\mathrm{L}, \mathrm{t})=\sqrt{R} \mathrm{E}_{\mathrm{F}}(\mathrm{L}, \mathrm{t})
\end{aligned}
$$

where:

$$
E_{F}(z, t)=\varepsilon_{F}(z) e^{-i\left(\omega t-k_{0} z\right)}
$$




$$
\begin{aligned}
& E_{B}(z, t)=\varepsilon_{B}(z) e^{-i\left(\omega t-k_{0}(L-z)\right)} \\
& p_{F}(z, t)=\rho_{F}(z) e^{-i\left(\omega t-k_{0} z\right)} \\
& p_{B}(z, t)=\rho_{B}(z) e^{-i\left(\omega t-k_{0}(L-z)\right)}
\end{aligned}
$$

and $\mathrm{L}$ is the thickness of the medium or the separation between the two parallel mirrors of the cavity. The subscripts $F$ and $B$ refer to the forward and backward fields inside the cavity, respectively. The manipulation of Eqs. (10, 15 and 17) gives the following differential equation:

$$
\frac{d \varepsilon_{F}(z)}{d z}=\frac{i \omega}{2 \epsilon_{0} C} \rho_{F}(z)
$$

Similarly from Eqs.(11), (16) and (18), we get:

$$
\frac{d \varepsilon_{B}(z)}{d z}=-\frac{i \omega}{2 \in_{0} C} \rho_{B}(z)
$$

Eqn.(7) can be rewritten for the forward and backward polarization amplitudes through Eqn.(3) as:

$$
\begin{aligned}
& \rho_{F}(z)=\frac{\Gamma e^{i \theta}}{1+\left[2 \eta\left|\varepsilon_{F}(z)\right|^{2} / A_{21}\right]} \varepsilon_{F}(z) \\
& \rho_{B}(z)=\frac{\Gamma e^{i \theta}}{1+2 \eta\left|\varepsilon_{B}(z)\right|^{2} / A_{21}} \varepsilon_{B}(z)
\end{aligned}
$$

where:

$$
\begin{gathered}
\Gamma=\frac{\gamma N}{\left[\left(\omega_{a}^{2}-\omega^{2}\right)^{2}+4 \beta^{2} \omega^{2}\right]^{1 / 2}} \\
\eta=\frac{|\mu / \hbar|^{2} \beta / 2}{\left(\omega_{a}-\omega\right)^{2}+\beta^{2}}
\end{gathered}
$$

To solve the forward field Eqn. (19) through Eqn. (21), we first neglect the nonlinear dependence of $\rho_{F}(z)$ on the field. Thus we get,

$$
\frac{d \varepsilon_{F}(z)}{d z}=\frac{i \omega}{2 \in_{0} C} \Gamma e^{i \theta} \varepsilon_{F}(z)
$$


The solution of this equation is given by:

$$
\varepsilon_{F}(z)=\varepsilon_{F}(0) e^{\frac{i \omega \Gamma}{2 \epsilon_{0} C}(\cos \theta+i \sin \theta) z}
$$

Substituting for $\left|\varepsilon_{F}(z)\right|^{2}$ from Eqn. (25) into Eqn. (19) after substituting for $\rho_{F}(z)$, we have,

$$
\frac{d \varepsilon_{F}(z)}{d z}=\frac{i \omega}{2 \in_{0} C} \frac{\Gamma e^{i \theta}}{1+\frac{2 \eta}{A_{21}}\left|\varepsilon_{F}(0)\right|^{2} e^{-\alpha_{0} z}} \varepsilon_{F}(z)
$$

where $\alpha_{0}=\frac{\omega \Gamma \sin \theta}{\in_{0} C}$ is the linear absorption coefficient of the medium.

The solution of Eqn. (26) is given by:

$$
\varepsilon_{F}(z)=\varepsilon_{F}(0) e^{-\frac{\alpha_{F} z}{2}} e^{i k_{F} z}
$$

Where

$$
\alpha_{F}=\frac{1}{z} \ln \left[\frac{e^{\alpha_{0} z}+\frac{2 \eta}{A_{21}}\left|\varepsilon_{F}(0)\right|^{2}}{1+\frac{2 \eta}{A_{21}}\left|\varepsilon_{F}(0)\right|^{2}}\right]
$$

and

$$
k_{F}=\frac{\cot \theta}{2 z} \ln \left[\frac{e^{\alpha_{0} z}+\frac{2 \eta}{A_{21}}\left|\varepsilon_{F}(0)\right|^{2}}{1+\frac{2 \eta}{A_{21}}\left|\varepsilon_{F}(0)\right|^{2}}\right]
$$

Therefore, the forward field $E_{F}(z, t)$, given by Eqn. (15), can be written as:

where,

$$
E_{F}(z, t)=\varepsilon_{F}(0) e^{-\frac{\alpha_{F} z}{2}} e^{-i\left(\omega t-K_{F} z\right)}
$$

$$
K_{F}=k_{0}+k_{F}
$$


Here $\alpha_{F}$ represents the absorption coefficient of the medium as a nonlinear function of the field. It varies with the distance that the field travels through the medium. This variation comes from the location variation of the field. On the other hand $K_{F}$ represents the propagation constant of the forward field. It varies also nonlinearly with the field. In the present work we will take only into consideration the nonlinearity coming from the absorption coefficient $\alpha_{F}$ and ignore the nonlinearity in $K_{F}$.

Since a sufficient number of reflected beams inside the cavity is required to obtain a feedback sufficient for creating bistability, the absorption coefficient should be sufficiently low. For instance let us consider a medium of absorption coefficient $\alpha$ and thickness $L$, placed inside a Fabry-Perot cavity its both mirrors are having a reflection coefficient $R$. Then, the number of effective reflected beams will be $1 /\left[1-\mathrm{Re}^{-\alpha L}\right]$. If $R=0.99$ then, $\alpha L$ should be equal to 0.095 to create ten reflected beams. Under this consideration, referring to Eqn. (28) and taking $\alpha_{0} \mathrm{z}$ being very small, the approximation $e^{\alpha_{0} z} \approx 1+\alpha_{0} z+\frac{1}{2}\left(\alpha_{0} z\right)^{2}$ gives:

$$
\alpha_{F} \approx \frac{1}{z} \ln \left[1+\frac{\alpha_{0} z+\frac{1}{2}\left(\alpha_{0} z\right)^{2}}{1+\frac{2 \eta}{A_{21}}\left|\varepsilon_{F}(0)\right|^{2}}\right]
$$

Using the Taylor series expansion $\ln (1+x) \approx x-\frac{x^{2}}{2}$, Eqn. (32), can be rewritten as:

$$
\alpha_{F} \approx \frac{\alpha_{0}+\frac{1}{2} \alpha_{0}^{2} z}{1+\frac{2 \eta}{A_{21}}\left|\varepsilon_{F}(0)\right|^{2}}-\frac{z}{2}\left[\frac{\alpha_{0}+\frac{1}{2} \alpha_{0}^{2} z}{1+\frac{2 \eta}{A_{21}}\left|\varepsilon_{F}(0)\right|^{2}}\right]^{2}
$$

For $\mathrm{z}=0$, we have:

$$
\alpha_{F}=\frac{\alpha_{0}}{1+\frac{2 \eta}{A_{21}}\left|\varepsilon_{F}(0)\right|^{2}}
$$


This form corresponds to the known formula representing the absorption coefficient of a nonlinear medium inside a cavity [14].

$$
\alpha=\frac{\alpha_{0}}{1+I_{C} / I_{S}}
$$

where $I_{C}$ is the intracavity intensity and $I_{S}$ is the saturation intensity of the atomic medium inside the cavity.

Thus, if $I_{C}=\frac{1}{2} \in_{0} C\left|\varepsilon_{F}(0)\right|^{2}$

$$
I_{S}=\frac{1}{4} \in_{0} C \frac{A_{21}}{\eta}
$$

Since we are interested only in studying the dependence of the absorption coefficient on the field intensity, it is desirable to take the average value of Eqn. (33) over the cavity length.

$$
\begin{aligned}
\alpha_{F_{\text {avg }}} & =\frac{1}{L} \int_{0}^{L} \alpha_{F} \cdot d z \\
\alpha_{F_{\text {avg }}} & =\frac{\alpha_{0}+\frac{1}{4} \alpha_{0}^{2} L}{1+\frac{I_{C}}{I_{S}}}-\frac{\alpha_{0}^{2} L\left(\frac{1}{2}+\frac{\alpha_{0} L}{3}+\frac{\alpha_{0}^{2} L^{2}}{16}\right)}{2\left(1+\frac{I_{C}}{I_{S}}\right)^{2}}
\end{aligned}
$$

In the same manner to the previous steps, Eqn. (20) can be solved to obtain the backward field function in the form:

$$
E_{B}(z, t)=\varepsilon_{B}(L) e^{-\frac{\alpha_{B} \cdot(L-z)}{2}} e^{-i\left(\omega t-K_{B} \cdot(L-z)\right)}
$$

where

$$
\begin{aligned}
& \alpha_{B}=\frac{1}{(L-z)} \ln \left[\frac{e^{\alpha_{0}(L-z)}+\frac{2 \eta}{A_{21}}\left|\varepsilon_{B}(L)\right|^{2}}{1+\frac{2 \eta}{A_{21}}\left|\varepsilon_{B}(L)\right|^{2}}\right] \\
& K_{B}=k_{0}+k_{B}
\end{aligned}
$$

and 


$$
k_{B}=\frac{\cot \theta}{2(L-z)} \ln \left[\frac{e^{\alpha_{0}(L-z)}+\frac{2 \eta}{A_{21}}\left|\varepsilon_{B}(L)\right|^{2}}{1+\frac{2 \eta}{A_{21}}\left|\varepsilon_{B}(L)\right|^{2}}\right]
$$

Applying now the boundary conditions (12-14) on the forward and backward fields (30) and (39) respectively, we get the following expression for the transmitted field from the cavity $E_{T}(L, t)$ :

$$
E_{T}(L, t)=\frac{T E_{I n}(0, t) e^{-\frac{\alpha_{F} L}{2}} e^{i K_{F} \cdot L}}{1-R e^{-\left(\frac{\alpha_{F}+\alpha_{B}}{2}\right) L} e^{i\left[K_{F}+K_{B}\right] . L}}
$$

For high value of $R$, we can assume that $\alpha_{F}=\alpha_{B}=\alpha$ and $K_{F}=K_{B}=K$

[16]. Therefore, the intensity of the field transmitted from the cavity is given by:

$$
\begin{aligned}
& I_{T}=\left|E_{T}(L, t)\right|^{2} \\
& I_{T}=I_{I n} \frac{T^{2} e^{-\alpha L}}{1+R^{2} e^{-2 \alpha L}-2 \operatorname{Re}^{-\alpha L} \cos \delta}
\end{aligned}
$$

This is the "Airy formula", where

$$
\delta=2 K L
$$

and $I_{I n}=\left|E_{I n}(0, t)\right|^{2}$ is the intensity of the field incident on the cavity. The resultant field amplitude inside the cavity $E_{C}(z, t)$ due to the forward field $E_{F}(z, t)$ and the backward field $E_{B}(z, t)$ is given by:

and

$$
\begin{aligned}
& E_{C}(z, t)=E_{F}(z, t)+E_{B}(z, t) \\
& E_{F}(z, t)=\frac{\sqrt{T} E_{I n}(0, t) e^{\frac{-\alpha z}{2}} e^{-i(\omega t-K z)}}{1-\operatorname{Re}^{-\alpha L} e^{-i \delta}}
\end{aligned}
$$

$E_{B}(z, t)=\frac{\sqrt{T} \sqrt{R} E_{I n}(0, t) e^{\frac{-\alpha L}{2}} e^{i K L} e^{\frac{-\alpha(L-z)}{2}} e^{-i(\omega t-K(L-z))} e^{i \phi}}{1-\operatorname{Re}^{-\alpha . L} e^{-i \delta}}$ 
where $\phi$ is the phase gained by reflection of the backward wave with respect to the forward wave. Thus, the intracavity intensity is given by:

$$
I_{C}=I_{I n} T\left[\frac{e^{-\alpha z}+R e^{-\alpha(2 L-z)}+2 \sqrt{R} e^{-\alpha L} \cos [2 K(L-z)] \cos \phi}{1+R^{2} e^{-2 \alpha L}-2 R e^{-\alpha L} \cos \delta}\right]
$$

Eqn. (49) shows that the intracavity intensity is a sum of the intensities of the forward and backward fields plus a third term arising from the interference between the forward and backward fields. The presence of the third term induces a standing wave inside the cavity.

The standing wave can be eliminated when the phase $\phi$ gained by reflection of the backward wave with respect to the forward wave takes values $(2 m-1) \pi / 2$, where $m$ is an integer number. In this case we get a nonstanding wave inside the cavity with intensity given by the sum of the first two terms of Eqn. (49).

The intensity inside the cavity shows fluctuations due to $\cos 2 K(L-z)$. Since the maximum intensity is the dominant factor affecting the bistability phenomena we will take $\cos 2 K(L-z)=1$.

Therefore, Eqn. (49) can be rewritten as:

$$
I_{C}=I_{I n} T\left[\frac{e^{-\alpha z}+R e^{-\alpha(2 L-z)}+2 \sqrt{R} e^{-\alpha L} \cos \phi}{1+R^{2} e^{-2 \alpha L}-2 \operatorname{Re}^{-\alpha L} \cos \delta}\right]
$$

Since the present work deals with the study of the bistability phenomena arisen in the output intensity, the average intensity inside the cavity $I_{C_{\text {avg }}}$ has to be considered. This gives at resonance, i.e. $\delta=2 n \pi$ with $n$ an integer number, the following expression:

$$
I_{C_{\text {avg }}}=I_{I n} T\left[\frac{\frac{1}{\alpha L}\left(1-e^{-\alpha L}\right)\left(1+\mathrm{Re}^{-\alpha L}\right)+2 \sqrt{R} \mathrm{e}^{-\alpha L} \cos \phi}{\left(1-\mathrm{Re}^{-\alpha L}\right)^{2}}\right]
$$

\subsection{Computation.}

From Eqns. (38, 44 and 51), assuming a value for $\alpha_{0} L$ and given values for $I_{C} / I_{S}$, corresponding values for $I_{I n} / I_{S}$ are calculated from Eqn. (51). 
Substituting in Eqn. (44), after dividing its both sides by $I_{S}$, for the values of $I_{C} / I_{S}$ and the corresponding values of $I_{I n} / I_{S}, I_{T} / I_{S}$ can be calculated. The calculations are carried out for $\mathrm{R}=0.99$ and $\alpha_{0} L=0.1,0.2,0.3,0.4$, and 0.5 . $I_{C} / I_{S}$ takes values from 0 to 50 in steps of 0.05 leading to variation in the nonlinear absorption coefficient $\alpha$ from $\alpha_{0}$ to $0.02 \alpha_{0}$. Figures (1 to 6) show the behavior of the relative intensities of $I_{I n} / I_{S}$ against $I_{C} / I_{S}$, and $I_{I n} / I_{S}$ against $I_{T} / I_{S}$ for $\phi=0, \pi / 2$ and $\pi$, respectively.

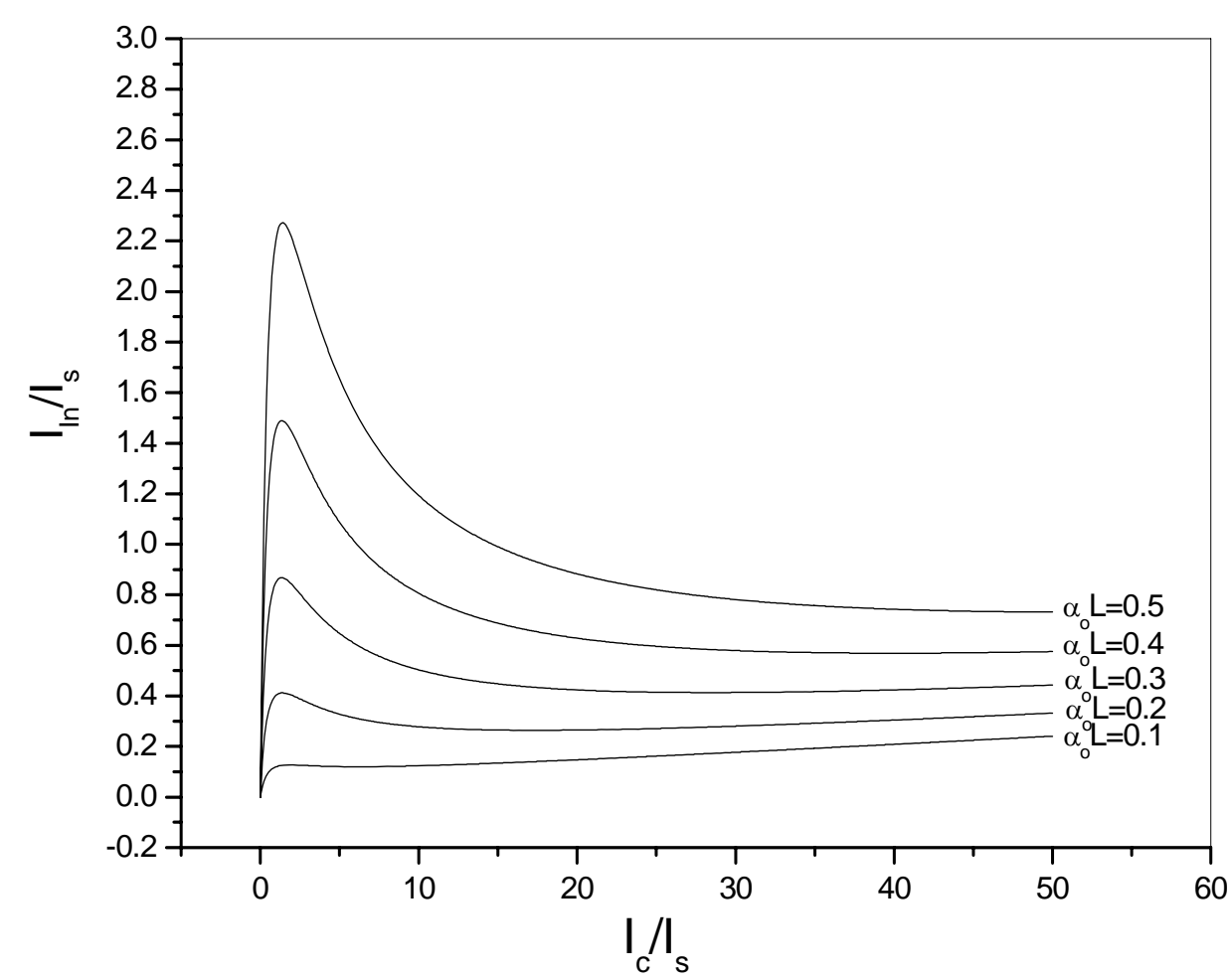

Fig. (1): The dependence of $I_{I n} / I_{s}$ on $I_{c} / I_{s}$ calculated in case of standing wave for $R=0.99$ and different values of $\alpha_{o} L$, and $\varphi=0$ 


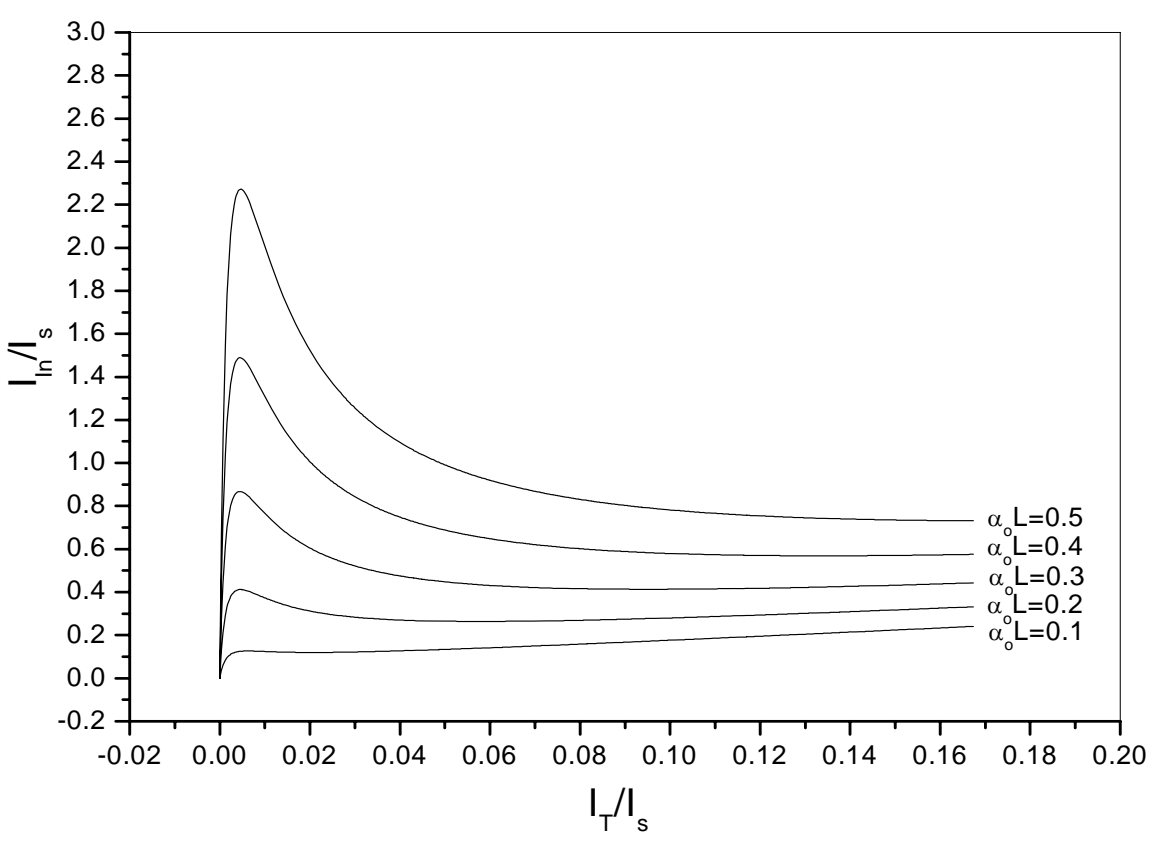

Fig. (2): The dependence of $I_{I n} / I_{s}$ on $I_{T} / I_{s}$ calculated in case of standing wave for $R=0.99$ and different values of $\alpha_{o} L$, and $\varphi=0$.

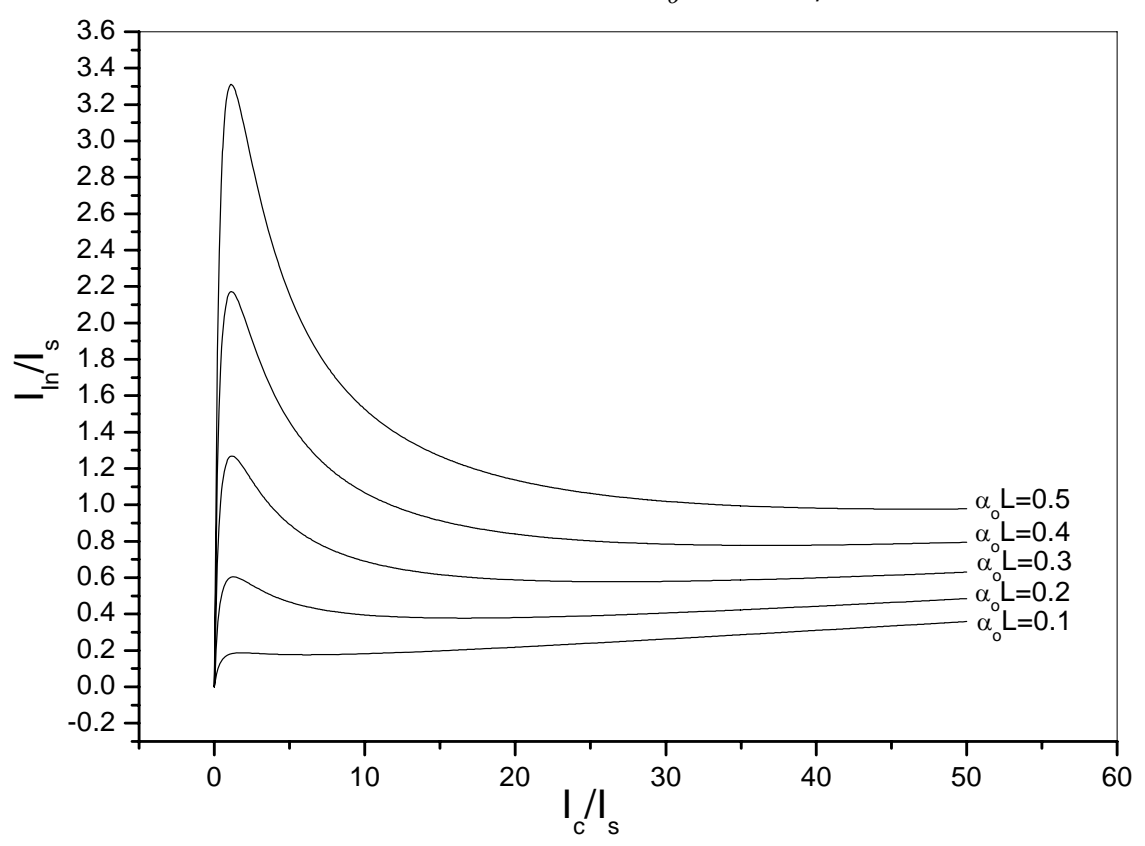

Fig. (3): The dependence of $I_{I n} / I_{s}$ on $I_{c} / I_{s}$ calculated in case of nonstanding wave for $R=0.99$ and different values of $\alpha_{o} L$ and $\varphi=\pi / 2$. 


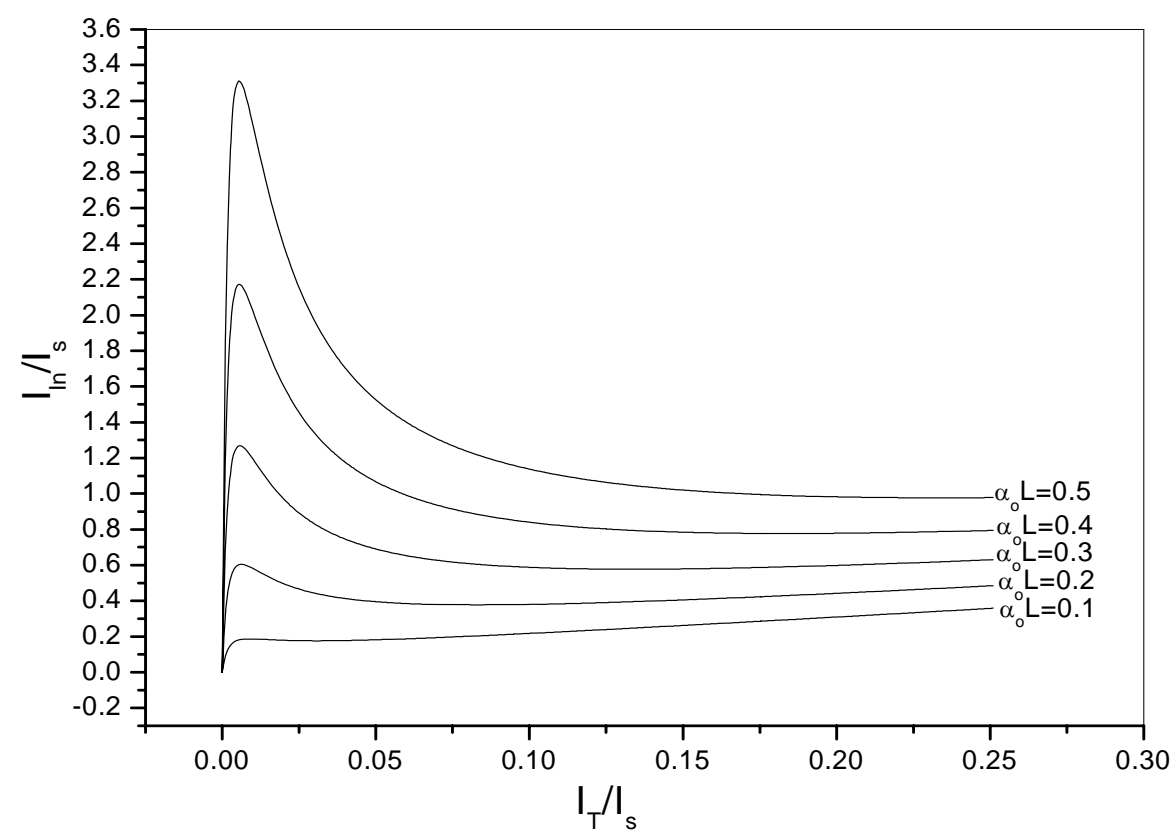

Fig. (4): The dependence of $I_{I n} / I_{s}$ on $I_{T} / I_{S}$ calculated in case of nonstanding wave for $R=0.99$ and different values of $\alpha_{o} L$ and $\varphi=\pi / 2$.

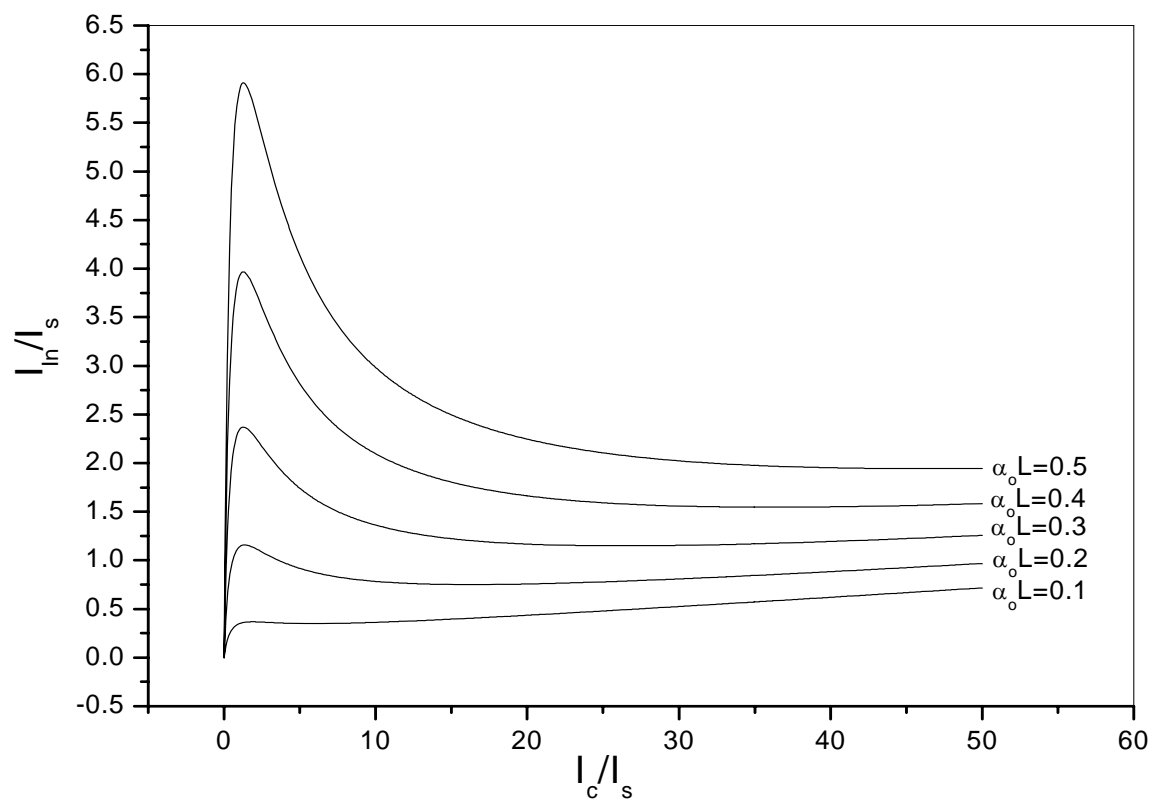

Fig.(5): The dependence of $I_{I n} / I_{s}$ on $I_{c} / I_{s}$ calculated in the case of standing wave for $R=0.99$ and different values of $\alpha_{o} L, \varphi=\pi$. 


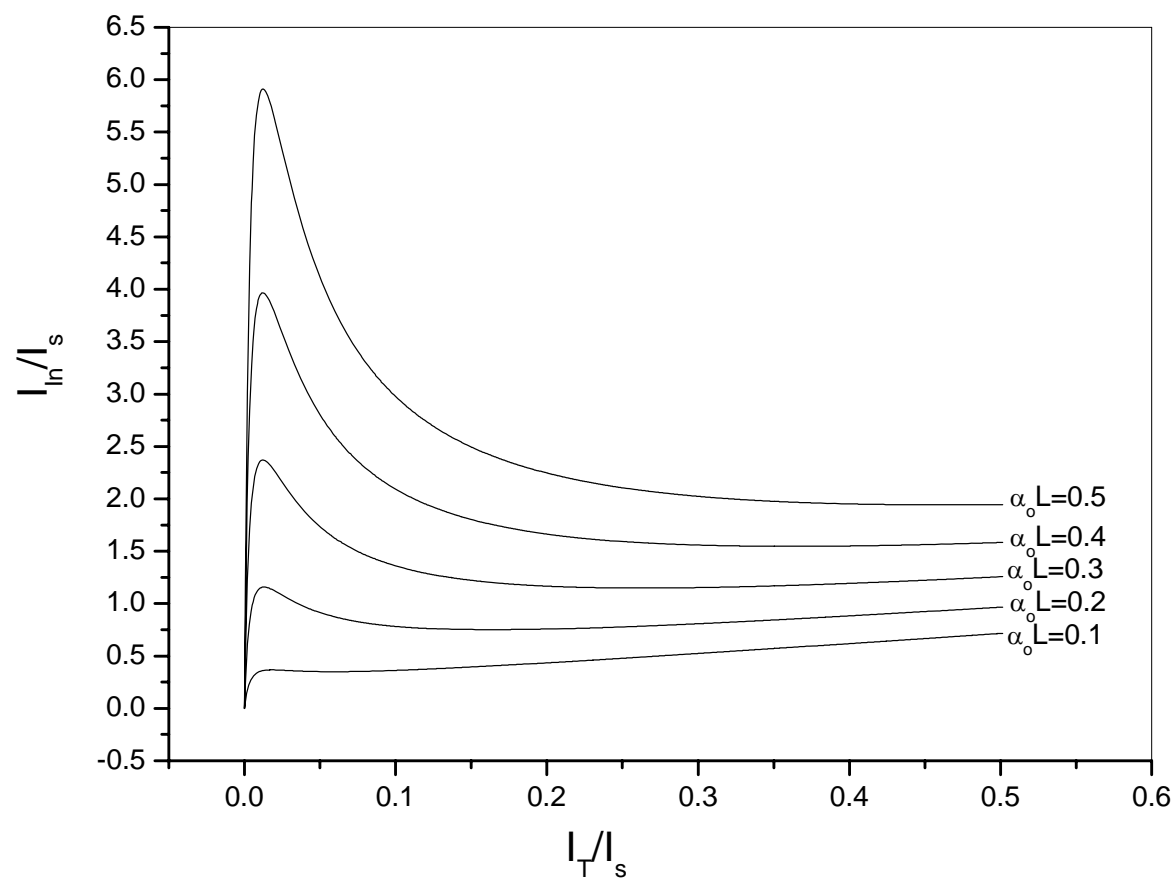

Fig.(6): The dependence of $I_{\text {Iin }} / I_{s}$ on $I_{T} / I_{s}$ calculated in the case of standing wave for $R=0.99$ and different values of $\alpha_{o} L,(\varphi=\pi)$.

\subsection{Discussion:}

Equation (38) shows that the absorption coefficient of the medium is a nonlinear function of the intracavity intensity. This justifies the first factor required for initiating bistability. This nonlinearity comes from the dependence of the absorption coefficient, in our case, on the population difference which varies nonlinearly with the deriving field. The boundary conditions (12-14) provide the feedback, i.e. the second factor for initiating bistability.

In both cases, standing wave and nonstanding wave, the bistability phenomena appear in both behaviors of $I_{I n}$ against $I_{C}$ and $I_{I n}$ against $I_{T}$, as shown in Figs.(1 to 6 ). The phase $\phi$ gained by reflection of the backward field with respect to the forward field inside the cavity plays an important role in defining the deriving intensity $I_{I n}$ required to initiate bistability. The input intensity required to initiate bistability, for a given value of $\alpha_{0} L$, has a minimum value when $\phi=2 m \pi$. There is an interconnection between $R, \phi, I_{I n}$ 
and $\alpha L$ leading, under certain conditions, to the occurrence of bistability. So for instance small values of $I_{I n}$ leads to a small $I_{C}$ value which in turn leads to a weak dependence of $\alpha$ on $I_{C}$. On the other hand an increase of $I_{I n}$ over a certain value leads to an increase of $I_{C}$ such that the nonlinear absorption coefficient $\alpha$ tends to be weak dependent on $I_{C}$ and thus to interrupt the bistability. The increase in the absorptivity $\alpha L$ implies an increase in the input intensity to initiate bistability. Therefore, in treatment of Fabry-Perot bistability, it is convenient to work in the limit of small optical absorptivity $\alpha L$ and small mirror transmission $\mathrm{T}$.

\section{Conclusion:}

In both cases of building standing and nonstanding waves inside the cavity, the bistability phenomena appear between the incident and transmitted intensities. The phase gained by reflection plays an important role in defining the deriving field intensity required to initiate bistability. Increasing both the absorptivity of the medium and transmission of the cavity mirrors implies an increase in the input intensity to initiate bistability.

\section{References.}

1. Peng-Wang Zhai, Xue-Mei Su and Jin-Yue Gao, Physics Letters A 289, 27 (2001).

2. O. Guillot-Noel, Ph.Goldner and M. Popova, Journal of Luminescence 119120, 478 (2006).

3. P. D. Maker, R. W. Terhune, M. Nisenhoff, and C. M. Savage, Phys. Rev. Lett 8 , 21 (1962).

4. José García Solé, Maria de la Ramírez, Airán Ródenas, Daniel Jaque, Lui Bausá, Marco Bettinelli, Adolfo Speghini, Enrico Cavalli and Lioudmila Ivleva, Journal of Luminescence 119-120, 314 (2006).

5. T. W Carr. and Erneux, Europ. J.Phys.D 17, 67 (2001).

6. David Yemele, Pierre Kisito Talla and Timoleon Korfane, J. Phys. D: Appl. Phys. 36, 1429 (2003).

7. Quintero R., Torres and Thakur M., Appl. Phys. Lett. 66 (11), 1310 (1995).

8. Andy Brown, Amitabn Joshi and Min Xiao., Appl. Phys. Lett., 83 (7), 1301 (2003).

9. K. An, B. A. Sones, C. Fang-Yen, R. R. Desari and M. S. Feld, Opt. Lett., 22 (17), 1433 (1997).

10. K. Koyonov, N. Parair, F. Bertrand, R. El Bermil. and P. Dansas, J. Opt. A: Pure Appl. Opt. 3, 26 (2001). 
11. V. Koryukin. and Paul Mandal, J. Opt. B: Quan. Semiclass. Opt. 4, 27 (2002).

12. McCall S.L., Phys. Rev., A 9, 1515 (1974).

13. Savage C.M and Carmichael H.J., Quan. Elec., IEEE J. 24, 1495, (1988).

14. M. M. EL-Nicklawy, A.F.Hassan, S.M.M.Salman and A.Abdel-Aty, Optics and Laser Technology, 34, 363 (2002).

15. M.M.EL-Nicklawy, A.F.Hassan, S.M.M.Salman and A. Abdel-Aty, Optics and Laser Technology 37, 363 (2005).

16. M. M. EL-Nicklawy, A. F. Hassan, A. T. Matar, A. A. Hemeda and A. I. Ali, Egypt. J. Solids, 29, 241 (2006).

17. Anthony E.Siegman, "Lasers", Oxford University Press. 110 (1986).

18. Peter W. Milonni and Joseph H.Eberly, "Lasers", John Wiley and Sons. Inc., pp. 213-215 (1988). 